\begin{tabular}{ccc}
\hline & International Journal of Engineering \&Technology, $7(3.12)(2018) 531-535$ \\
SPC & International Journal of Engineering \& Technology \\
Website: $w$ ww.sciencepubco.com/index.php/IJET & Research paper \\
\hline
\end{tabular}

\title{
Energy Consumption Analysis in WSN Based on Node Placement Strategies
}

\author{
R.Shanmugavalli ${ }^{1 *}$, P.Subashini $^{2}$ \\ ${ }^{1}$ Research Scholar, ${ }^{2}$ Professor, ${ }^{1,2}$ Department of Computer Science, \\ Avinashilingam Institute for Home Science and Higher Education for Women, Coimbatore, India \\ *Corresponding Author Email: ${ }^{1}$ rsvalli1294@gmail.com, ${ }^{2}$ parthasarathysubashini@gmail.com
}

\begin{abstract}
Wireless Sensor Networks (WSNs) is a collection of devices and sensor nodes connected with wireless network and communicate with one another via radio signals. Sensor in WSN is an autonomous (self-configuring) device used to sense the light, heat, motion, moisture and pressure etc that communicate with their neighbor nodes. Node placement is a technique that places the nodes effectively in the specified network environment. In WSN basically, wireless sensor network includes different topologies namely star, point-to-point, ring, bus, mesh and hybrid. In recent years, research has been carried out on different node placement strategies and produced different results based on its performance that includes power distribution and energy consumption of sensors. Energy consumption and network lifetime are considered to be the critical issues as the nodes are powered by the batteries which have finite energy reservoirs. In this paper, three different node placements namely Random, Uniform and Grid with respect to AODV (Ad hoc On-Demand Distance Vector) protocol is evaluated in order to analyze the energy factor during wireless communication. The performance metrics used to measure the analysis are Energy Consumption Average Jitter, Average End-to-End Delay, Average Throughput and Average Packet Delivery Ratio. The comparison results suggests that Grid node placement performs well in grid scenarios and shows best for specific performance metrics.
\end{abstract}

Keywords: Wireless Sensor Networks, Node Placement, AODV Protocol, Performance Evaluation, Energy Consumption, Ad hoc Network.

\section{Introduction}

A wireless sensor network (WSN) is a collection of cooperative sensor devices to support more applications in different environments such as monitoring, environmental conditions, medical diagnostics, structural monitoring and so on. It is also considered as self configuring autonomous device. In [1], authors defined that WSNs consist of set of mobile wireless nodes communicating without the support of any pre-existing fixed infrastructure. Sensor nodes are placed in different environmental location and follows various network topologies using wireless networks. The nodes gather more information via other neighbors and information are forwarded to other base stations or end users. The Wireless Sensor Networks includes different node placement method and each placement will provide different end results.

One of the most important issues in wireless sensor networks is energy efficiency. Wireless Sensor Networks majorly affects energy constrains because nodes depends on battery and there is a need to minimize the total energy consumption of the nodes. And also Wireless Sensor nodes service lifetime depends on energy consumption of the communication subsystem therefore channel capacity being used for data transmission. Hence energy consumption is mainly used for increasing the node life time, by consuming lowest energy. In [2], authors analyzed performance and compared physical and application layers of different mobility models with CBR traffic load.

This proposed work has considered three routing protocols for mobile ad hoc networks and evaluated the energy factor, based on routing power and residual energy [3]. In the proposed work, with

respect to node placement strategies such as Random, Grid and Uniform, experiments where consumed on different increased number of nodes. Different metrics for energy analysis is evaluated based on physical layer parameters and packet analysis is done based on application layer parameters.

To test the competence and effectiveness of all the three node placement strategies, comparison is done by means of different increased number of nodes and using AODV (Ad hoc On-demand Distance Vector) routing protocol and CBR (Constant Bit Rate) connections. Constant Bit Rate is an encoding method that keeps the bit rate in same size. It is used in this traffic model to transfer the data with very high data processing and fast data transmission based on Ad hoc On-Demand Distance Vector (AODV), a reactive protocol. The organization of the paper is as follows: Section I discusses the introduction of this paper. Section II briefly describes the three types of Wireless Sensor Network node placements strategy. Section III discusses the experimental set up and results analysis with discussion and Section IV defines the performance analysis on simulation results and conclusion.

\section{WSN Node Placements}

Wireless Sensor Networks includes different topologies and placement strategies, and structures available for its physical environment establishment. In this paper three types of node placements strategies are analyzed and compared with metrics.

\subsection{Random Node Placement}

Copyright $\odot 2018$ Authors. This is an open access article distributed under the Creative Commons Attribution License, which permits unrestricted use, distribution, and reproduction in any medium, provided the original work is properly cited. 
Wireless sensor nodes are placed randomly with in fixed physical terrain area. It uses multiple sensor nodes placed randomly within the fixed physical terrain area as shown in Fig 1. Each node will be placed in different individual places, without following any order. This placement strategy creates node density in which congestion occurs easily. It is a common node placement strategy basically used in different environment and nodes are placed randomly without following same distances.

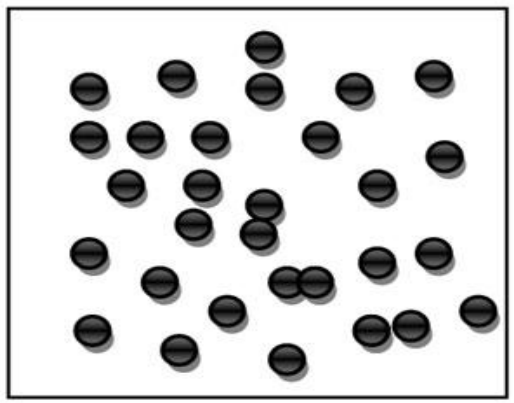

Fig. 1: Random Node Placement Strategy

\subsection{Grid Node Placement}

Grid node placement places the start node at $(0,0)$ format and the nodes are placed in a grid with each node a grid-unit away from its other neighbor nodes as shown in Fig 2. In this placement strategy, a node is placed by grid order and maintains same distance with each node.

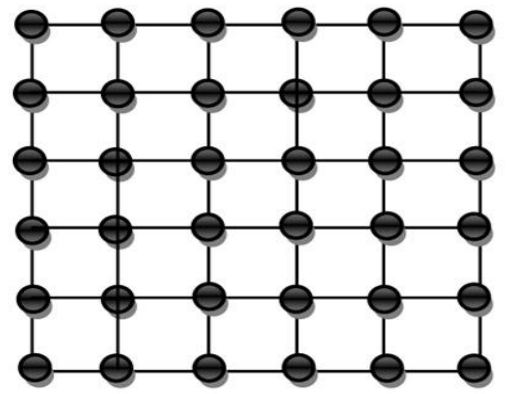

Fig. 2: Grid Node Placement Strategy

The sensor nodes are placed in individual cell with an order. This placement must also be specified numerically, with the unit in meters depending on the value of coordinate system. In [4], authors proposes three node placement models concluding that energy can be efficiently utilized in WSNs, with the grid placement Grid placement mainly reduces the overall energy consumed by the network further increasing the network lifetime.

\subsection{Uniform Node Placement}

This node placement is same as random node placement method, with small difference where placement of nodes are divided in each individual cell and placed without any order as shown in Fig 3. In [5], authors defined that uniform node placement is based on the number of nodes in the simulated physical terrain area and divided into a number of cells where each node is placed randomly. This generates random strategy but with uniform density of nodes.

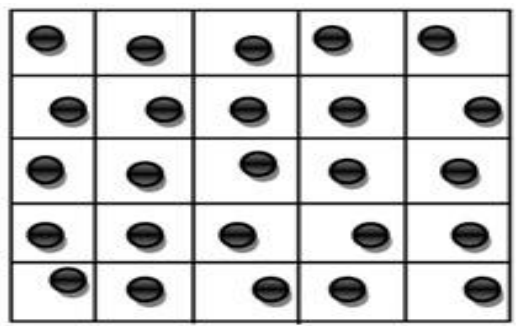

Fig. 3: Uniform Node Placement Strategy

\section{Experimental Setup and Results Analysis}

This paper work compares random, uniform and grid node placement strategies evaluated with three different set of nodes such as 10,20 , and 30. Data packets is successfully transmitted to destination node in support with Ad hoc On-Demand Distance Vector (AODV) routing protocol. All the three node placements is done on same terrain size $(800 * 800)$ and also follows the same process. All sender nodes will be directly connected with the single receiver node at a time, and nodes are directly connected with CBR data traffic model. The Fig 4 shows the experimental scenarios of different node placement with CBR data traffic connection.

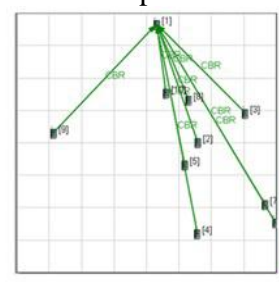

Random

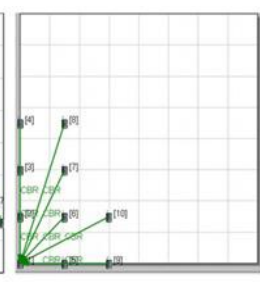

Grid

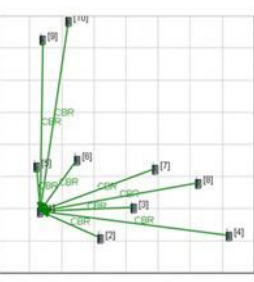

Uniform
Fig. 4: Node Placement Scenarios

Wireless Sensor Network platform creates the radio signals and time sensor node sends and receives data packets with other neighbor nodes using radio signals. During run time, radio signals are created and green color mark is given to indicate the same in Fig. 5. The radio signals successfully transfers the data packets with respect to AODV routing protocols and it allows sensor nodes to obtain routes quickly for new destinations, and does not require nodes to maintain routes to destinations that are not in active communication. From Patil V.P [6] it is understood that in the AODV routing protocol, the moving speed will be maximum during end-to-end delay.

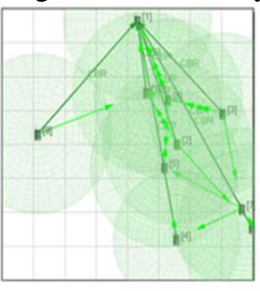

Random

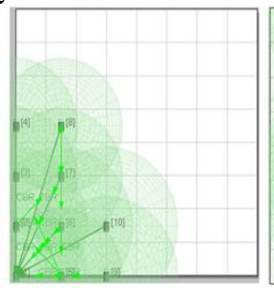

Grid

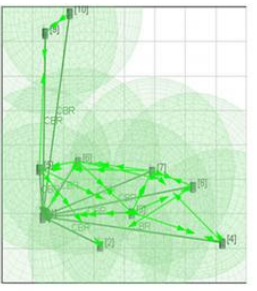

Uniform
Fig. 5: Node Placements with Animated View

\subsection{Node Configuration Details}

Each network nodes configuration is analyzed with the performance metrics in physical layer and application layer as which are shown in Table 1. The simulation setup includes different wireless network parameters given in table 1 .

Table 1: Node Configuration Details

\begin{tabular}{|c|c|}
\hline PARAMETERS & VALUES \\
\hline Number of Nodes & 10,20 and 30 \\
\hline Terrain Size & $800 * 800$ \\
\hline Node Placement & Random, Uniform, and Grid \\
\hline Traffic Source & CBR \\
\hline No.of CBR Connections & 9,19 , and 29 \\
\hline Energy Model & Mica Motes \\
\hline Battery Model & Linear Model \\
\hline Mobility Model & Random Way Point \\
\hline Routing Protocol & AODV \\
\hline Radio Type & $802.11 \mathrm{~b}$ \\
\hline MAC Protocol & 802.11 \\
\hline Network Protocol & IPv4 \\
\hline Antenna Model & Omidirectional \\
\hline Simulation Time & 30 Seconds \\
\hline Performance Metrics in Appli- & Average Jitter, Average End-to-End \\
\hline
\end{tabular}




\begin{tabular}{|l|l|}
\hline cation Layer & $\begin{array}{l}\text { Delay, Average Throughput, and Pack- } \\
\text { et Delivery Ratio }\end{array}$ \\
\hline $\begin{array}{l}\text { Performance Metrics in Physi- } \\
\text { cal Layer }\end{array}$ & $\begin{array}{l}\text { Energy consumed in transmit mode } \\
\text { Energy consumed in receive mode } \\
\text { Energy consumed in idle mode }\end{array}$ \\
\hline
\end{tabular}

\subsection{Experimental Analysis}

The energy consumption of the sensor nodes depends on the battery that is analyzed based on two layers 1.Physical Layer, 2.Application Layer. The application layer parameter is also analyzed to signify the performance of Grid node strategy.

\subsubsection{Energy Consumption Analysis Parameters - Phys- ical Layer}

Physical layer mainly represents for energy conservation. In WSN there are different energy conservation parameters: Energy Consumption in Transmit mode, Energy Consumption in Receive Mode and Energy Consumption in Idle Mode that are involved in physical layer. Each parameter represents a various level of energy consumption.

\section{a) Energy Consumed in Transmit Mode}

It is an energy consumed by battery power during the transmission of data packets from source node to destination node.

\section{b) Consumed in Receive Mode}

This is an energy consumed by battery power for receiving the packets at the destination node is called energy consumed in receive mode.

\section{c) Consumed in Idle Mode}

The energy consumed by battery power in the idle stage of the network is termed as energy consumed in idle mode.

Table 2: Performance Analysis of Physical Layer

\begin{tabular}{|c|c|c|c|c|}
\hline No of Nodes & Parameters & Random & Grid & Uniform \\
\hline \multirow{3}{*}{10} & Energy Consumed in Transmit Mode (mjoule) & 0.006 & 0.004 & 0.006 \\
\hline & Energy Consumed in Receive Mode (mjoule) & 0.019 & 0.007 & 0.009 \\
\hline & Energy Consumed in Idle Mode (mjoule) & 0.12 & 0.122 & 0.121 \\
\hline \multirow{3}{*}{20} & Energy Consumed in Transmit Mode (mjoule) & 0.008 & 0.008 & 0.017 \\
\hline & Energy Consumed in Receive Mode (mjoule) & 0.026 & 0.021 & 0.029 \\
\hline & Energy Consumed in Idle Mode (mjoule) & 0.112 & 0.114 & 0.105 \\
\hline \multirow{3}{*}{30} & Energy Consumed in Transmit Mode (mjoule) & 0.014 & 0.011 & 0.013 \\
\hline & Energy Consumed in Receive Mode (mjoule) & 0.052 & 0.045 & 0.052 \\
\hline & Energy Consumed in Idle Mode (mjoule) & 0.103 & 0.103 & 0.097 \\
\hline
\end{tabular}

\subsubsection{Packet Delivery Analysis Parameters - Application Layer}

\section{a) Average Jitter}

Jitter is the difference in the time arrival of the packets. The average jitter value is measured in seconds. Sensors transmit data packets to the destination node continuously and during that process a sensor node maintains the uniform distance between numbers of packets. But, when the packets reaches destination node, the distance between each packets widely varies due to various network problems that includes network congestion, improper queuing, configuration errors, that delay leads packet to transmission time in between each packet. Rohit Sangwan et al. has also discussed that in transmission scenario various packets take different amount of time in reaching destination [7].

\section{b) Average End-to-End Delay}

The average time taken by a source node to transfer data packets to the destination node in the network is termed as average end-toend delay. In [8], author defined end to end delay parameter gives the total delay in time the data packets suffer while moving from source to destination across the network. There are four delay parameters such as processing delay, queuing delay, propagation delay and end system processing delay.

End-to-End Delay $=\mathrm{N}\left[\mathrm{d}_{\text {trans }}+\mathrm{d}_{\text {prop }}+\mathrm{d}_{\text {proc }}+\mathrm{d}_{\text {queue }}\right]$

Where,

$\mathrm{N}=$ Number of delays

$\mathrm{d}_{\text {trans }}=$ Transmission Delay

$\mathrm{d}_{\text {prop }}=$ Propagation Delay $\mathrm{d}_{\text {proc }}=$ Processing Delay

$\mathrm{d}_{\text {queue }}=$ Queuing Delay

\section{c) Average Throughput}

Throughput is defined as the number of packets transferred from one node (source) node to another node (destination) in a unit time. The mathematical average throughput is measured in number of bits per second (bits/sec). Throughput is, defined as the overall data packets delivered over the total simulation time [9]. The representation of throughput is denoted.

Throughput $\leq \frac{\text { RVIN }}{R T T}$

Where,

RWIN $=$ The TCP receive window .

RTT $=$ The Round-Trip Time for the path.

\section{d) Average Packet Delivery Ratio}

The packet delivery ratio is the ratio of the number of data packets created at the source node to the number of packets received by the destination node. The high number of bits transfer reveals the better system performance. Dharam et al. discussed that packet delivery ratio is calculated by dividing the number of packets revised by the destination through the number of packets originated by the application layer of the source [4]. The packet delivery ratio is an important metric in wireless sensor networks and defines the ratio of the entire data packets created by the source node [10]. The equation for calculating the PDR is represented as.

Average $P D R=\frac{S 1}{S 2}$ 
$\mathrm{S} 1=$ The total amount of data packets are generated in source nodes.

S2 = Number of data packets successfully send the destination node.

PDR $=$ Packet Delivery Ratio

Table 3: Performance Analysis of Application Layer

\begin{tabular}{|c|c|c|c|c|}
\hline $\begin{array}{l}\text { No of } \\
\text { Nodes }\end{array}$ & Parameters & Random & Grid & Uniform \\
\hline \multirow[t]{4}{*}{10} & Average Jitter (s) & 0.091 & 0.055 & 0.043 \\
\hline & $\begin{array}{c}\text { Average End-to-End } \\
\text { Delay (s) }\end{array}$ & 0.371 & 0.195 & 0.514 \\
\hline & $\begin{array}{l}\text { Average Throughput } \\
\text { (s) }\end{array}$ & 37477.5 & 38540.6 & 24290 \\
\hline & $\begin{array}{l}\text { Average Packet } \\
\text { Delivery Ratio }\end{array}$ & 8.68 & 9 & 5.655 \\
\hline \multirow{4}{*}{20} & Average Jitter (s) & 0.486 & 0.296 & 0.725 \\
\hline & $\begin{array}{c}\text { Average End-to-End } \\
\text { Delay (s) }\end{array}$ & 1.79 & 1.358 & 2.931 \\
\hline & $\begin{array}{c}\text { Average Throughput } \\
\text { (s) }\end{array}$ & 76753.15 & 80747 & 66447.45 \\
\hline & $\begin{array}{l}\text { Average Packet } \\
\text { Delivery Ratio }\end{array}$ & 8.68 & 18.771 & 15.58 \\
\hline \multirow{4}{*}{30} & Average Jitter (s) & 1.604 & 1.29 & 1.526 \\
\hline & $\begin{array}{c}\text { Average End-to-End } \\
\text { Delay (s) }\end{array}$ & 6.027 & 5.441 & 5.963 \\
\hline & $\begin{array}{l}\text { Average Throughput } \\
\text { (s) }\end{array}$ & 102815.4 & 107364 & 99180.07 \\
\hline & $\begin{array}{l}\text { Average Packet } \\
\text { Delivery Ratio }\end{array}$ & 24.159 & 25.519 & 23.963 \\
\hline
\end{tabular}

From the experiment it is understood that placing the nodes by following the Grid strategy will be more efficient in less consumption of energy there by leading to increase in the life time of the batteries.

\subsubsection{Discussion}

The observation of the experimental results portrayed in Fig. 6 it is identified that, Grid placement strategy gives better results, in terms of minimizing the energy consumption in transmit mode values when compared to random and uniform placement strategies. The average energy consumption in transmit mode in the random placement is 0.00894413, uniform placement is 0.01144467 , and grid placement is 0.0073573 . The average energy consumption is analyzed in which Grid node placement strategy scams to be more suitable.

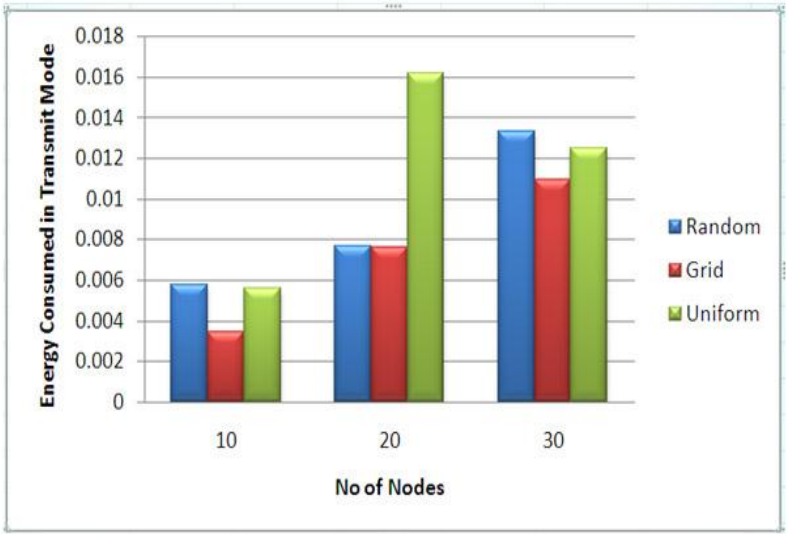

Fig. 6: Energy Consumed in Transmit Mode

From the observation of the experimental results portrayed in Fig. 7 it is identified that, Grid placement strategy gives better results, in terms of minimizing the energy consumption in idle mode values when compared to random and uniform placement strategies. The average energy consumption in transmit mode in random placement is 0.031786 , uniform placement is 0.029458 , and grid placement is 0.024088 . The average energy consumption is analyzed in which Grid node placement strategy gives better performance.

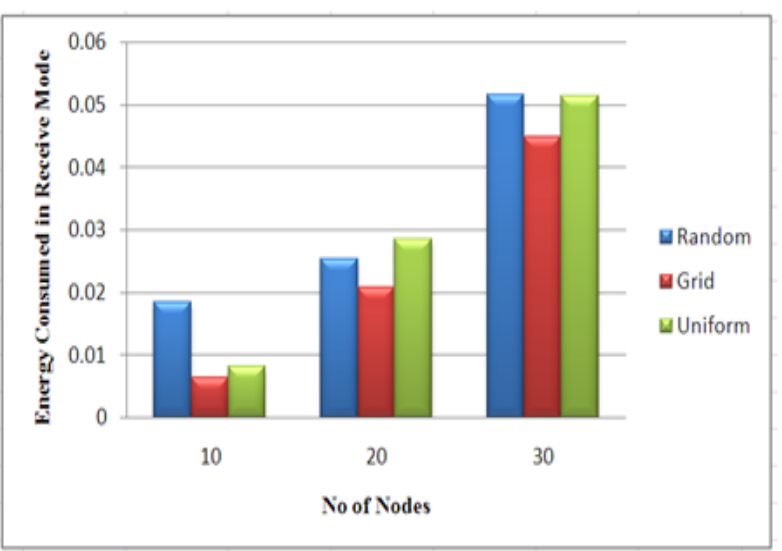

Fig. 7: Energy Consumed in Receive Mode

From the physical layer observation of experimental results portrayed in Fig. 8 it is identified that, random node placement strategy gives better results in 10 nodes when compared another two set of nodes. The uniform placement strategy gives better results, in terms of minimizing the energy consumption in idle mode values when compared to random and grid placement strategies in 20 and 30 nodes. The node size increases from 10 to 30 , in average energy consumption in idle mode in random is 0.72651333 , uniform is 0.76413333 , and the grid is 0.546358 . The energy consumption is analyzed to ensure the performance of best in grid node placement strategy.

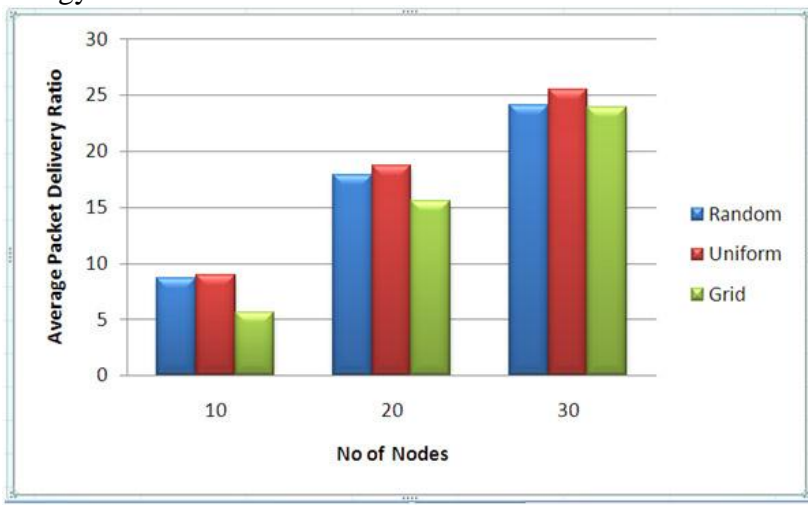

Fig. 8: Energy Consumed in Idle Mode

From the observation of the experimental results portrayed in Fig. 9 , it is identified that, uniform node placement strategy gives better results in 10 nodes when compared another two set of nodes. Grid placement strategy gives better results, in terms of minimizing the jitter values when compared to random and uniform placement strategies in 20 and 30 nodes. When the node size increases from 10 to 30 , the average jitter in random is 0.72651333 , uniform is 0.76413333 , and the grid is 0.546358 . The best average jitter performance is analyzed and it indicates that grid node placement strategy to be best. 


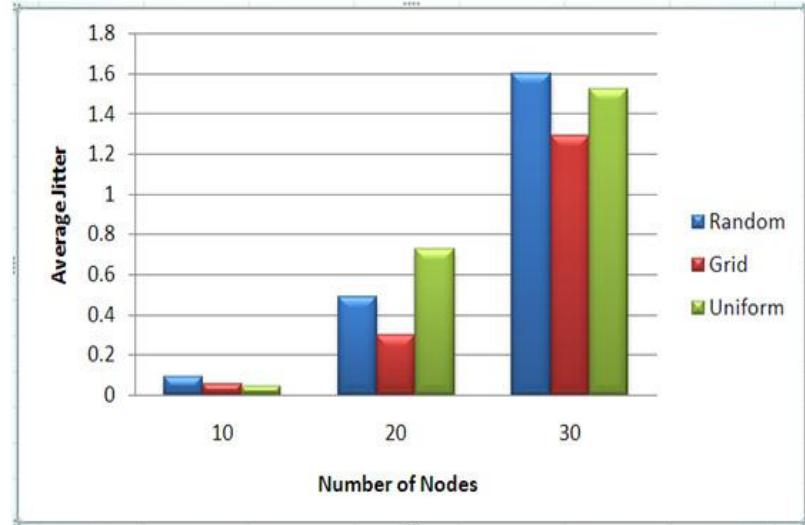

Fig. 9: Average Jitter

From the observation of the experimental results portrayed in Fig. 10 it is identified that, Grid placement strategy gives better results, in terms of minimizing the end-to-end delay values when compared to random and uniform placement strategies. The average end-to-end delay in random is 2.72881133 , uniform is 3.13557933 , and grid is 2.33113167 . The best average end-to-end delay performance is analyzed and it indicates that grid node placement strategy to be best.

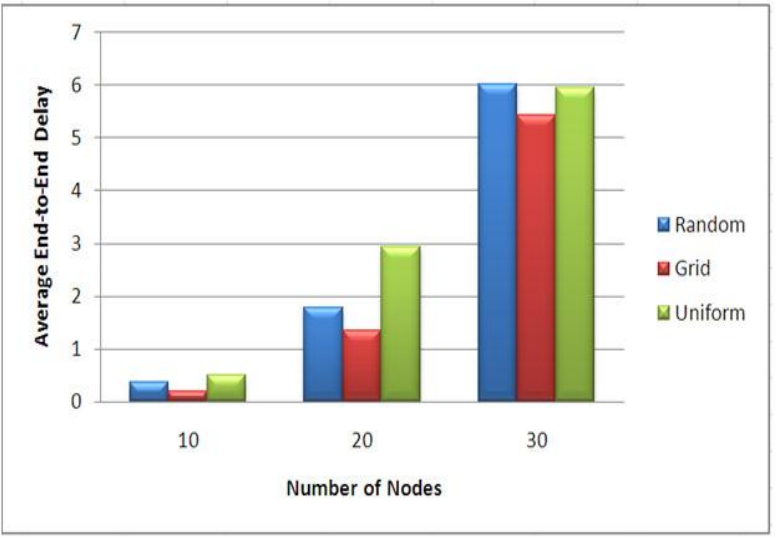

Fig. 10: Average End-to-End Delay

From the observation of the experimental results portrayed in Fig. 11 it is identified that, Grid placement strategy gives better results, in terms of maximizing the throughput values when compared to random and uniform placement strategies. The average throughput in random is 72348.6833, uniform is 63305.84, and grid is 75550.5333. The best average throughput performance is analyzed and it indicates that grid node placement strategy to be best.

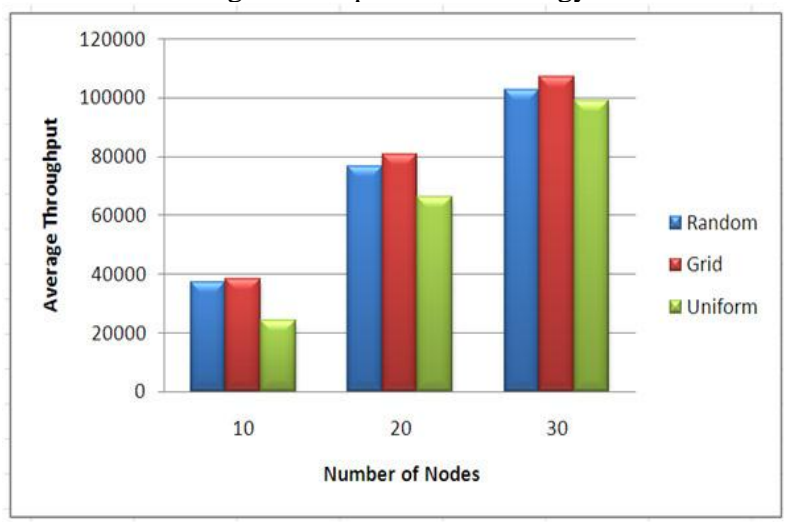

Fig. 11: Average Throughput

From the observation of the experimental results portrayed in Fig. 12 it is identified that, Grid placement strategy gives better results, in terms of maximizing the packet delivery ratio values when compared to random and uniform placement strategies. The average packet delivery ratio in random is 16.9243057 , uniform is
15.06528 , and grid is 17.7629633 . Average packet delivery ratio performance results are analyzed where uniform node placement strategy proves better.

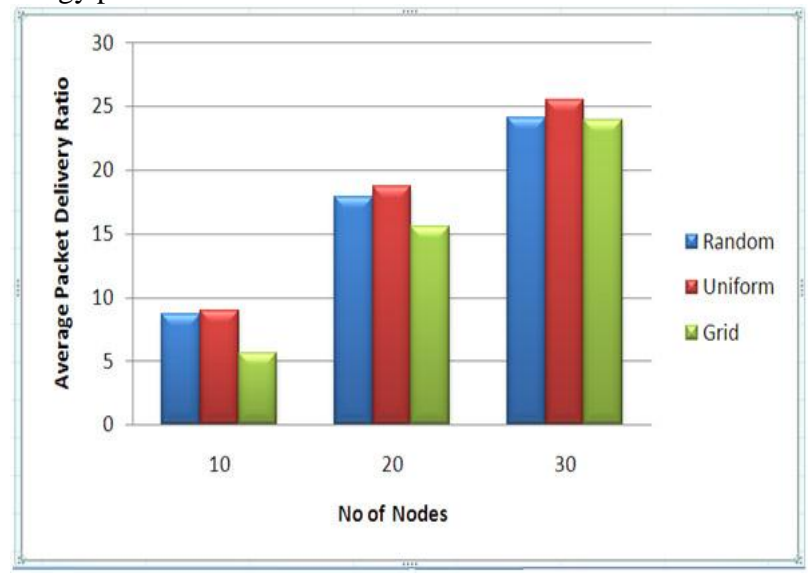

Fig. 12: Average Packet Delivery Ratio

\section{Conclusion}

In this paper energy consumption of node placement strategies in wireless sensor networks is investigated. It is observed that energy balancing is an important optimization objective in wireless sensor networks, as energy consumption during process depends on computing ability, limited battery and storage capacity. Here, three different node placement strategies are compared on the basics of their physical and application layer parameters on three different set of nodes. From the, observation of experimental the results it is identified that, Grid placement strategy gives better results when compared to random and uniform placement strategies.

\section{References}

[1] Tony Ducrocq, Michael Hauspie, Nathalie Mitton, Sara Pizzi, " On the impact of network topology on wireless sensor networks performances," Advanced Information Networking and Applications Workshops, June 2014

[2] Dharam Vir, Dr.. S.K.Agarwal, Dr.S.A.Imam, "Simulation of energy consumption analysis of multi-hop 802.11 wireless ad-hoc networks on reactive routing protocols," International Journal of Engineering Research and Applications, vol. 3, no. 1, pp. 1213-1218, January-February 2013.

[3] Dharam Vir, Dr.S.K.Agarwal, Dr.S.A.Imam, “A simulation study on node energy constraints of routing protocols of mobile ad hoc networks use of qualnet simulator", International Journal of Advanced Research in Electrical, Electronics and Instrumentation Engineering, vol. 1, no. 5, November 2012.

[4] Krishnakumar Y.Bendigeri, Jayashree D. Mallapur, "Multiple node placement strategies for efficient routing in wireless sensor networks", Wireless Sensor Networks, vol. 7, pp. 101-112, 2015.

[5] Dr.Vikram Singh, Jyoti Yadav, "Impact of random, uniform node placement and grid environment on the performance of routing protocols in MANET," International Journal on Recent Innovation Trends in computing and Communication, vol. 4, no. 7, pp. 349-354, July 2016.

[6] Patil V.P, “ Efficient AODV routing protocol for MANET with enhanced packet delivery ratio and minimized end to end delay," International Journal of Scientific and Research Publications, vol. 2, no. 8, August 2012.

[7] Rohit Sangwan, Dr. Manoj Duhan, Sachin Dahiya, "Energy consumption analysis of ad hoc routing protocols for different energy models in MANET", IOSR Journal of Electronics and Communication Engineering, vol. 6, no. 4, pp. 85-55, May-June. 2013.

[8] Duhimi. Abd. Latif, Saqib Hakak, F. Anwar, M.K.Alam, "Effect of 3 key factors on average end to end delay in Manet",

[9] Pankaj Rohal, Ruchika Dhivya, Prashant Dhivya, " Study and analysis of throughput, delay and packet delivery ratio in MANET for topology based routing protocols (AODV, DSR and DSDV)," International Journal for Advanced Research in Engineering and Technology, vol. 1, no. II, march 2013.

[10] R.Shanmugavalli, P.Subashini, "A study on node placement strategies in wireless sensor networks" International Journal of Theoretical and Applied Sciences, vol. 10, no. 1, pp. 135-142, February 2018. 